\title{
SCORE-IT (Selecting Core Outcomes for Randomised Effectiveness trials In Type 2 diabetes): a systematic review of registered trials
}

Nicola L. Harman ${ }^{1 *}$, Rebecca James ${ }^{1}$, John Wilding ${ }^{2}$, Paula R. Williamson ${ }^{1}$ and on behalf of the SCORE-IT study team

\begin{abstract}
Background: Outcomes measured in clinical trials should be meaningful to patients, healthcare professionals and researchers, yet there is heterogeneity in the outcomes used across trials. This inconsistency impacts on the ability to compare findings and may mean that the results have little importance to healthcare professionals and the patients that they care for. The aim of the present study is to review the outcomes used in registered trials of therapies for type 2 diabetes mellitus as the first step in the development of a core outcome set for effectiveness trials in type 2 diabetes.

Methods: A systematic review of clinicaltrials.gov entries was completed for randomised, open (actively recruiting or in follow-up period), phase 3 and 4 trials of type 2 diabetes mellitus in adults. Trials of the treatment of diabetes complications, co-morbidities, prevention and surgery were excluded. Each trial was screened for eligibility and outcomes extracted from the primary and secondary outcomes data fields and free text study information. The outcomes were recorded verbatim and classified into core outcome domains according to the COMET taxonomy.

Results: A total of 354 trial registrations were reviewed for eligibility and 138 trials included. In total, 1444 outcomes were extracted with a median of eight outcomes per trial (range $=1-60$ ). Outcomes were categorised into 30 different outcome domains according to the COMET taxonomy, but no single domain or outcome was measured in $100 \%$ of trials. The majority of trials (88\%) included outcomes in the 'metabolism and nutrition' domain, such as lipids and lipoproteins (21\%), HbA1c (18\%), hypoglycaemia (14\%), fasting plasma/blood glucose (11\%), glycaemic variability (8\%), postprandial response (8\%) and insulin sensitivity (5\%). Only 10\% of trials included one or more patient reported outcomes; of these, 29\% included the Diabetes Treatment Satisfaction Questionnaire.
\end{abstract}

Conclusions: There is marked heterogeneity in the outcomes measured in registered therapeutic intervention trials for type 2 diabetes. The use of an agreed set of core outcomes will improve the consistency of reporting in clinical trials for type 2 diabetes.

Trial registration: The core outcome set study, of which this is a part, is registered in the COMET database, http:// www.comet-initiative.org/studies/details/956. Registered on 24 January 2017.

Keywords: Core outcome set, Systematic review, Type 2 diabetes

\footnotetext{
* Correspondence: n.harman@liv.ac.uk

${ }^{1}$ Department of Biostatistics, Institute of Translational Medicine, University of

Liverpool, Liverpool L69 3GL, UK

Full list of author information is available at the end of the article
} 


\section{Background (150-200)}

Type 2 diabetes mellitus accounts for over 90\% of all diabetes. It is characterised by abnormal glucose metabolism brought about by resistance to insulin action and an inadequate compensatory insulin secretory response $[1,2]$. The resulting hyperglycaemia, if left untreated, can lead to both macrovascular and microvascular complications which may be further exacerbated by obesity, elevated blood pressure and dyslipidaemia that are also often associated with type 2 diabetes mellitus [3].

Systematic reviews of glucose lowering treatments for type 2 diabetes have identified inconsistency in the outcomes measured and reported and while many routinely report glycated haemoglobin, other measures of glycaemic control and outcomes relating to hypoglycaemia, mortality, diabetes-related complications and quality of life are less frequently reported, if at all [3-8]. The heterogeneity in the outcomes used may impact on the translatability of trials into benefits for patients [9, 10]. The World Health Organization (WHO) International Classification of Functioning, Disability and Health (ICF) core set for diabetes mellitus contains 85 second level categories; 28 of these are included in the brief ICF core set that the ICF state can be used for the assessment of patients with diabetes participating in a clinical trial $[11,12]$. However, not only is it impractical to measure all 28 outcomes in the brief ICF core set in all trials, there is also an issue that it just includes outcomes related to function. Using only the brief ICF core set in clinical trials could mean that other outcomes important to patients and healthcare professionals are not measured.

One suggestion to improve the relevance and consistency of trial outcomes includes the development of a core outcome set (COS) that represents the minimum set of outcomes that should be measured and reported in any clinical trial for a given condition, in this case type 2 diabetes [13-15]. To ensure that no COS for trials of type 2 diabetes existed or was in development by another group, a review of entries in the Core Outcome Measures in Effectiveness Trials (COMET) initiative database was completed before commencing this project ((http://www.comet-initiative.org/), on 21 October 2016 and again before manuscript submission on 14 September 2017). No published or ongoing COS for the treatment of type 2 diabetes without co-morbidity was identified (Additional file 1).

Here we aim to describe the outcomes used in trials, currently recruiting, that evaluate therapeutic interventions for type 2 diabetes, registered in a large international public clinical trial registry, as the first step in the development of a COS for type 2 diabetes [15].

\section{Methods}

\section{Search strategy}

On 20 October 2016, the ClinicalTrials.gov database (www.clinicaltrials.gov) was searched using the following search terms: Type 2 diabetes; Type II diabetes; noninsulin dependent diabetes; Open studies; Interventional studies; Phase 3, 4; Studies received from 10/11/2007.

In the context of the clinicaltrials.gov registry, an 'open' study is one that is currently recruiting participants or will be recruiting participants in the future.

Clinicaltrials.gov was chosen as this registry allows outcomes to be easily identified and extracted and was the main source of trials in a previous study using trial registries to identify outcomes [7]. Trials registered before 10 November 2007 have been reported elsewhere [7].

\section{Eligibility criteria}

Phase 3 and 4 trials of therapeutic interventions for patients with type 2 diabetes were included. Trials were excluded if they met any of the following criteria: phase 1 and 2 trials (including entries listed as phase 2/phase $3)$; prevention trials; trials of treatment for diabetic foot ulcers, diabetic retinopathy or for diabetic nephropathy; trials of bariatric surgery; and trials of treatment for any other co-morbidity including non-alcoholic fatty liver disease and cardiovascular disease (trials assessing cardiovascular safety of glucose lowering drugs are eligible for inclusion). When trials were registered more than once, only the initial registration was included.

\section{Assessment of trial eligibility}

$\mathrm{NH}$ and RJ reviewed the first 40 trials together with full discussion about inclusion and exclusion of trials and outcome extraction. A further $5 \%$ of trials was then randomly selected and independently reviewed in parallel by the reviewers to ensure consistency. Where disagreement was noted, the reviewers discussed the study before reaching a decision. No study required third reviewer arbitration.

\section{Data extraction}

Data on study characteristics was extracted by $\mathrm{NH}$ that included trial phase, region, design, type of intervention (pharmaceutical, nutritional, educational/lifestyle or device) and duration of follow-up. Data on outcomes listed in the clinicaltrials.gov protocol registration entry were extracted by $\mathrm{NH}$ and RJ from the specific outcomes fields and from the study information free text. Where composite outcomes were used, all component outcomes were included. Where an outcome was reported in terms of the measurement instrument used, for example a particular questionnaire, the instrument was reviewed and outcomes extracted.

\section{Outcome classification}

$\mathrm{NH}$ categorised each outcome according to the COMET taxonomy of core domains [submitted for publication]. This taxonomy comprises 38 domains under five areas 
(death, physiological/clinical, life impact, resource use and adverse events). Functional outcomes were also categorised according to the ICF top level domains (http://www.who.int/classifications/icf/en/). A random check of categorisation was completed on $30 \%$ of outcomes by JW, discrepancies were resolved through consensus and discussion with a third reviewer (PRW) where necessary.

\section{Results}

\section{Search results and study characteristics}

The search returned 675 entries in the clinicaltrials.gov database; after duplicates were removed, 354 trials were screened for eligibility, of which 138 were included (trial registration numbers of included trials are available in Additional file 2). The flow of included trials is shown in Fig. 1.

Of the 138 eligible trials, 127 (92\%) were trials of drug interventions with the remainder evaluating educational or lifestyle $(4 \%)$, nutritional $(2 \%)$ or device (1\%) interventions. The majority (65\%) were phase 4 trials with $\leq 200$ participants (median $=135$, range $=12-5000)$ and follow-up of $\leq 6$ months (median $=24$ weeks, range $=$ 0-364 weeks). Characteristics of included trials are described in Table 1.

\section{Classification of trial outcomes COMET taxonomy}

A total of 1444 individual outcomes were extracted with a median of eight outcomes per trial (range $=1-60$ ). Each outcome was reviewed and categorised using the COMET taxonomy (Table 2).

The most frequently included domain was 'metabolism and nutrition' with $87 \%$ of trials measuring one or more outcomes in this domain and 92 (67\%) trials including an outcome from this domain as their primary outcome. The key outcomes included in 'metabolism and nutrition' were: outcomes related to lipids and lipoproteins (21\%); HbA1c (18\%); hypoglycaemia (14\%); fasting plasma/blood glucose (11\%); glycaemic variability $(8 \%)$; postprandial response (8\%); and insulin sensitivity (5\%). The remaining $21 \%$ of outcomes were varied and included markers of oxidative and nitrosative stress, gut hormones, energy expenditure and other non-specific metabolic markers.

Nearly half of the studies (47\%) included outcomes categorised as 'general outcomes' (outcomes that affect the whole body and cannot be attributed to a certain body system) which included outcomes related to body weight $(42 \%)$, adiposity $(17 \%)$, other anthropometric measures $(11 \%)$, clinical chemistry not attributed to one

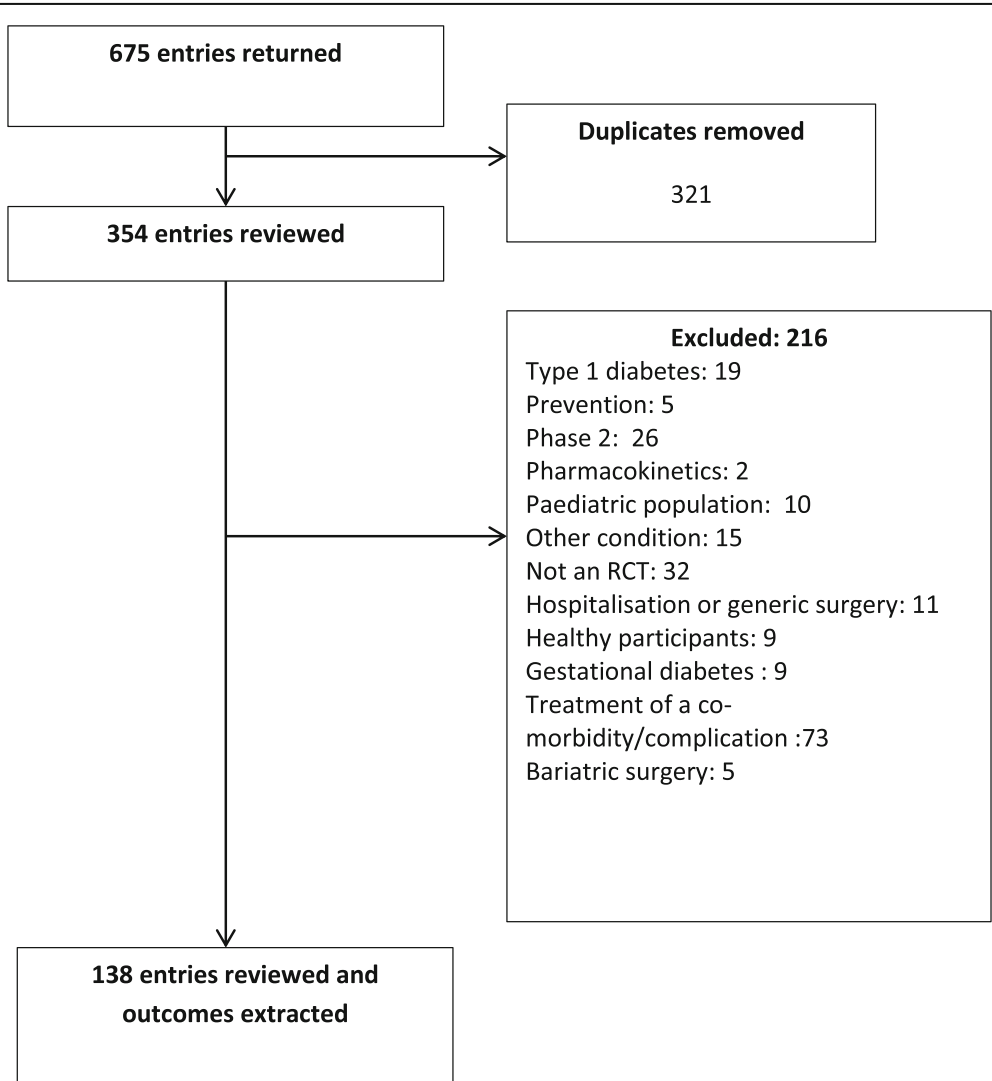

Fig. 1 Flow of included trials 
Table 1 Description of included trials

\begin{tabular}{|c|c|}
\hline & n (\%) \\
\hline \multicolumn{2}{|l|}{ Year } \\
\hline 2009 & $1(1)$ \\
\hline 2010 & $0(0)$ \\
\hline 2011 & $2(1)$ \\
\hline 2012 & $3(2)$ \\
\hline 2013 & $6(4)$ \\
\hline 2014 & $21(15)$ \\
\hline 2015 & $49(36)$ \\
\hline 2016 & $56(41)$ \\
\hline \multicolumn{2}{|l|}{ Phase } \\
\hline 3 & $48(35)$ \\
\hline 4 & $90(65)$ \\
\hline Planned enrolment (median and range) & $135(12-5000)$ \\
\hline \multicolumn{2}{|l|}{ Region of work ${ }^{a}$} \\
\hline Asia & $55(40)$ \\
\hline Europe & $45(33)$ \\
\hline North America & $46(33)$ \\
\hline South America & $8(6)$ \\
\hline Africa & $6(4)$ \\
\hline Central America & $4(3)$ \\
\hline Australia & $1(1)$ \\
\hline Not reported & $6(4)$ \\
\hline \multicolumn{2}{|l|}{ Trial design } \\
\hline Parallel & $125(91)$ \\
\hline Crossover & $11(8)$ \\
\hline Other & $2(1)$ \\
\hline \multicolumn{2}{|l|}{ Type of intervention } \\
\hline Drug & $137(92)$ \\
\hline Placebo & $83(60)$ \\
\hline Active drug & $36(26)$ \\
\hline Usual care & $1(1)$ \\
\hline Other & $7(5)$ \\
\hline Education or lifestyle & $3(2)$ \\
\hline Nutrition & $6(4)$ \\
\hline Device & $2(1)$ \\
\hline Duration of follow-up (median and range) ${ }^{b}$ & $24(0-364)$ weeks \\
\hline
\end{tabular}

${ }^{a}$ Number exceeds total as a number of studies were conducted across multiple geographical areas

${ }^{5} 0$ weeks $=<24-h$ follow-up $(n=3)$

particular body function or system (11\%), physical activity $(5 \%)$, fatigue $(3 \%)$ and non-specific pain $(3 \%)$. The remaining $10 \%$ of outcomes in the 'general outcomes' category included vital signs, non-specific patient reported outcomes (those with no detail provided in the clinicaltrials.gov entry other than 'patient-reported outcome'), general health, smoking status, morbidity and global effectiveness.

\section{Use of patient-reported outcome measures}

Fourteen (10\%) studies listed one or more patientreported outcome measures (PROMs). Twenty-three PROMs were identified which measured 68 outcomes (Table 3). The use of PROMs was varied and of the 23 PROMs, $87 \%$ were used in only one study. The most frequently used PROM was the Diabetes Treatment Satisfaction Questionnaire used by four (29\%) of the studies reporting PROMs.

\section{ICF core set and outcomes used in registered trials}

Of the 1444 individual outcomes, 80 (5.5\%) did not fit with any of the ICF categories. These outcomes included unspecified adverse events $(\mathrm{n}=44)$, treatment preference or satisfaction $(n=5)$, mortality $(n=2)$, pharmacokinetics $(\mathrm{n}=1)$ and general physiological or laboratory measures $(n=27)$. Ten categories in the ICF brief set and an additional 46 categories in the ICF full core set were not associated with any outcomes being measured in the trials. The breakdown of outcomes according to the ICF core set is provided in Additional files 3 and 4.

\section{Discussion}

There is heterogeneity in the outcomes used across registered open trials for type 2 diabetes. While some outcomes are commonly measured and are expected in trials that aim to treat hyperglycaemia, there is no consensus on which outcomes should be routinely measured and reported, with no single outcome or outcome domain being measured in all trials.

Reaney et al. have recently reviewed PROMs used in published phase 3 type 2 diabetes mellitus trials of GLP-1 receptor agonists, novel insulins, SGLT-2 inhibitors and DPP-4 inhibitors [16]. The identified PROMs in the included studies were mixed and varied compared to those identified in the present review, with overlap of only four measurement instruments (DTSQ, EQ5D, SF-36 and HFS-11 worry scale). The diabetes treatment satisfaction questionnaire (DTSQ) was the most frequently used PROM in both the review by Reaney et al. and in the present study which may be due to the recommendations made by the WHO to encourage psychological wellbeing in patients with diabetes [17]. In the present study, only $10 \%$ of trials included a PROM; this is comparable with the study by Barsdorf et al. in 2012 who found that only $7.5 \%$ of phase 3 pharmaceutical interventions for type 2 diabetes, registered with clinical trials.gov, included a PROM [18]. Gandhi et al. [7] considered patient important outcomes in registered trials, described as outcomes that affect the way patients feel, function or survive [8]. In 
Table 2 Summary of outcomes categorised according to the COMET taxonomy

\begin{tabular}{|c|c|c|c|c|}
\hline Core area & Core domains & $\begin{array}{l}\text { Trials including one or more } \\
\text { outcome in core domain }(n(\%))\end{array}$ & $\begin{array}{l}\text { Outcomes included in } \\
\text { core domain }(\mathrm{n}(\%))\end{array}$ & $\begin{array}{l}\text { Trials including as a } \\
\text { primary outcome }^{a}(n)\end{array}$ \\
\hline Death & Mortality/survival & $3(2.2)$ & $3(0.2)$ & 0 \\
\hline \multirow{24}{*}{$\begin{array}{l}\text { Physiological/ } \\
\text { clinical }\end{array}$} & Blood and lymphatic system outcomes & $9(6.5)$ & $19(1.3)$ & 1 \\
\hline & Cardiac outcomes & $20(14.5)$ & $56(3.9)$ & 9 \\
\hline & Congenital, familial and genetic outcomes & $0(0)$ & $0(0)$ & \\
\hline & Endocrine outcomes & $31(22.5)$ & $50(3.5)$ & 7 \\
\hline & Ear and labyrinth outcomes & $0(0)$ & $0(0)$ & 0 \\
\hline & Eye outcomes & $2(1.4)$ & $2(0.1)$ & 0 \\
\hline & Gastrointestinal outcomes & $5(3.6)$ & $20(1.4)$ & 2 \\
\hline & General outcomes & $65(47.1)$ & $146(10.1)$ & 3 \\
\hline & Hepatobiliary outcomes & $12(8.7)$ & $25(1.7)$ & 3 \\
\hline & Immune system outcomes & $28(20.3)$ & $73(5.1)$ & 4 \\
\hline & Infection and infestation outcomes & $4(2.9)$ & $8(0.6)$ & 0 \\
\hline & Injury and poisoning outcomes & $0(0)$ & $0(0)$ & 0 \\
\hline & Metabolism and nutrition outcomes & $121(87.7)$ & $582(40.3)$ & 92 \\
\hline & Musculoskeletal and connective tissue outcomes & $2(1.4)$ & $2(0.1)$ & 1 \\
\hline & $\begin{array}{l}\text { Outcomes relating to neoplasms: benign, } \\
\text { malignant and unspecified (including cysts } \\
\text { and polyps) }\end{array}$ & $0(0)$ & $0(0)$ & 0 \\
\hline & Nervous system outcomes & $6(4.3)$ & $16(1.1)$ & 2 \\
\hline & Pregnancy, puerperium and perinatal outcomes & $0(0)$ & $0(0)$ & 0 \\
\hline & Renal and urinary outcomes & 27 (19.6) & $76(5.3)$ & 5 \\
\hline & Reproductive system and breast outcomes & $0(0)$ & $0(0)$ & 0 \\
\hline & Psychiatric outcomes & $2(1.4)$ & $2(0.1)$ & 0 \\
\hline & Respiratory, thoracic and mediastinal outcomes & $3(2.2)$ & $11(0.8)$ & 1 \\
\hline & Skin and subcutaneous tissue outcomes & $1(0.7)$ & $1(0.1)$ & 0 \\
\hline & Vascular outcomes & $51(37)$ & $134(9.3)$ & 13 \\
\hline & Physical functioning & $5(3.6)$ & $7(0.5)$ & 0 \\
\hline \multirow[t]{8}{*}{ Life impact } & Social functioning & $5(3.6)$ & $6(0.4)$ & 0 \\
\hline & Role functioning & $3(2.2)$ & $6(0.4)$ & 0 \\
\hline & Emotional functioning/wellbeing & $8(5.8)$ & $28(1.9)$ & 0 \\
\hline & Cognitive functioning & $2(1.4)$ & $22(1.5)$ & 0 \\
\hline & Global quality of life & $4(2.9)$ & $5(0.3)$ & 0 \\
\hline & Perceived health status & $4(2.9)$ & $4(0.3)$ & 0 \\
\hline & Delivery of care & $30(21.7)$ & $60(4.2)$ & 4 \\
\hline & Personal circumstance & $0(0)$ & $0(0)$ & 0 \\
\hline \multirow[t]{4}{*}{ Resource use } & Economic & $4(4)$ & $6(0.4)$ & 0 \\
\hline & Hospital & $3(2.2)$ & $4(0.3)$ & 0 \\
\hline & Need for intervention & $16(11.6)$ & $24(1.7)$ & 1 \\
\hline & Societal/carer burden & $0(0)$ & $0(0)$ & 0 \\
\hline $\begin{array}{l}\text { Adverse } \\
\text { events }\end{array}$ & Adverse events/effects & $33(23.9)$ & $46(3.2)$ & 5 \\
\hline
\end{tabular}

${ }^{\mathrm{a}}$ Some trials included more than one primary outcome

the present study, over half (51\%) of trials included one or more outcomes meeting this definition. However, this definition was not developed with input from patients with type 2 diabetes and so may not truly reflect outcomes of treatment that they consider to be the most important. 


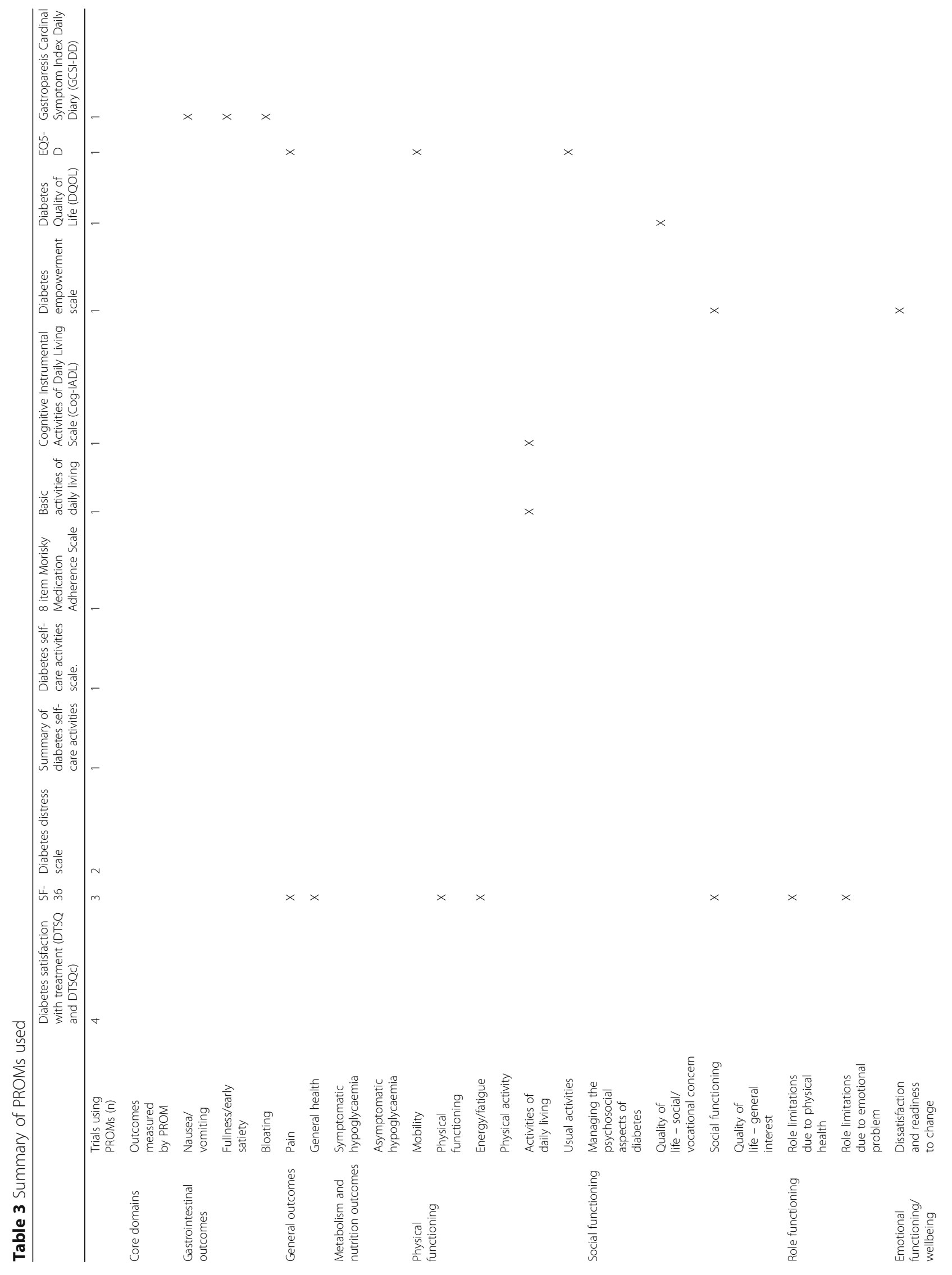




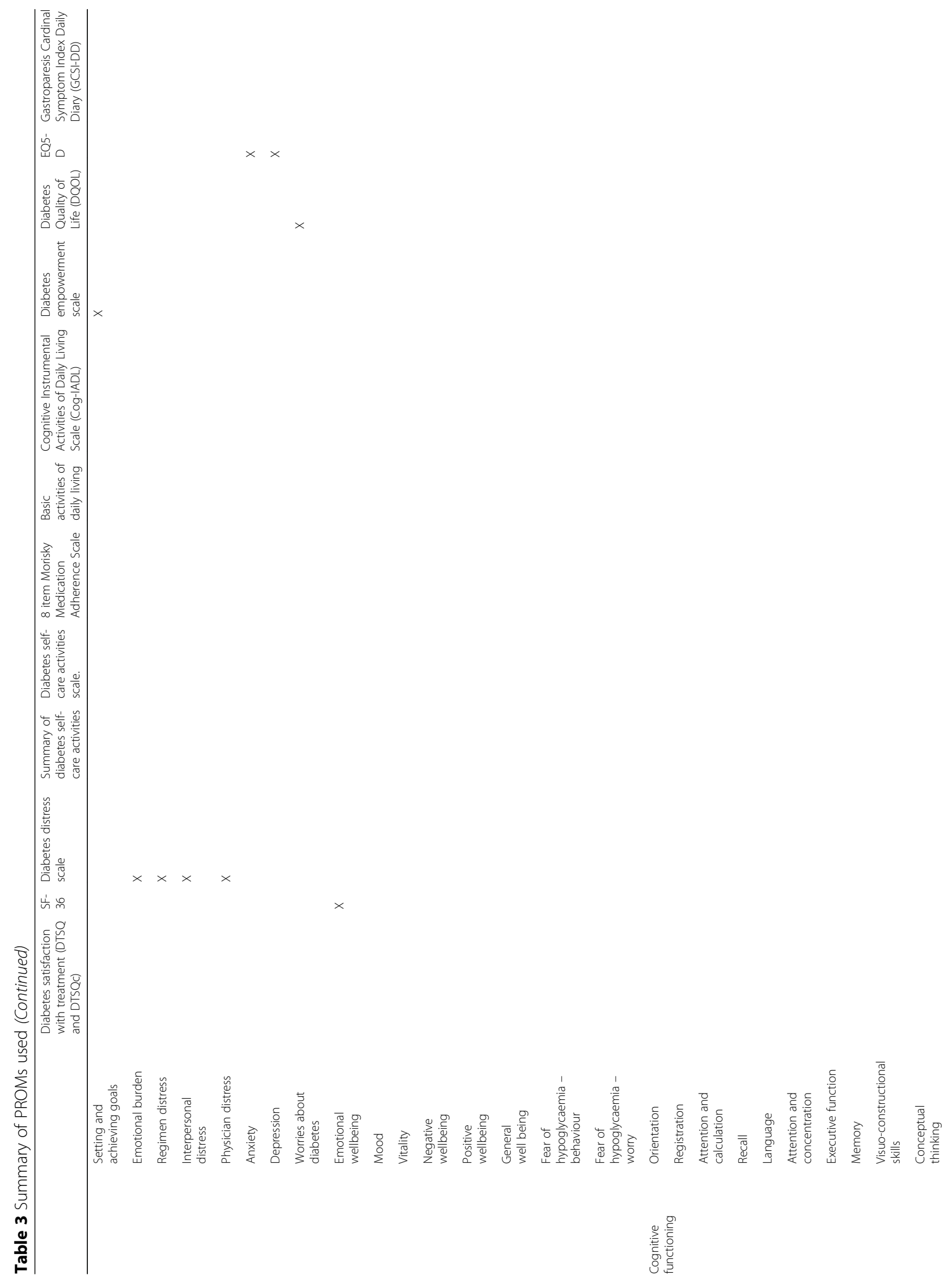




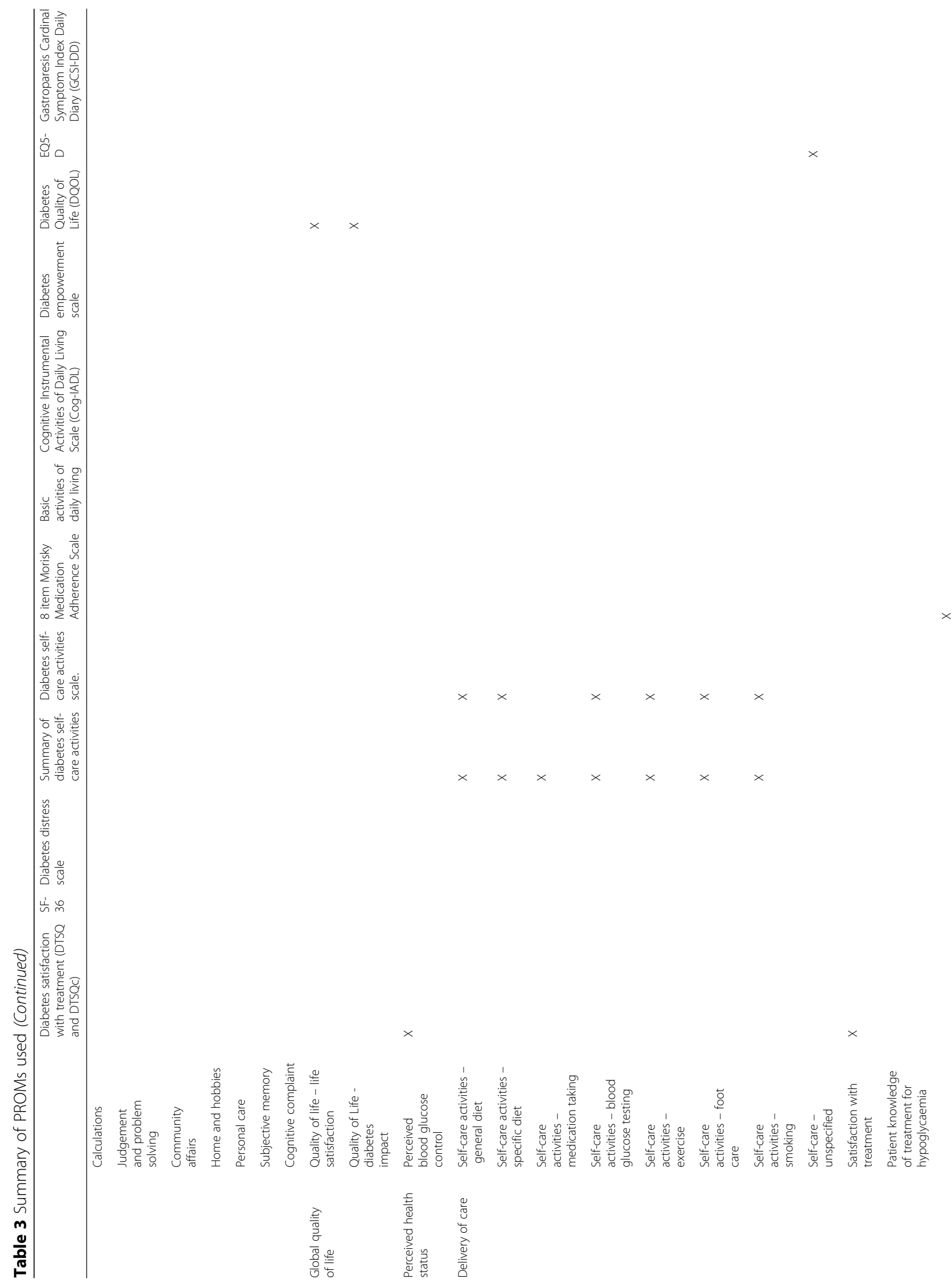




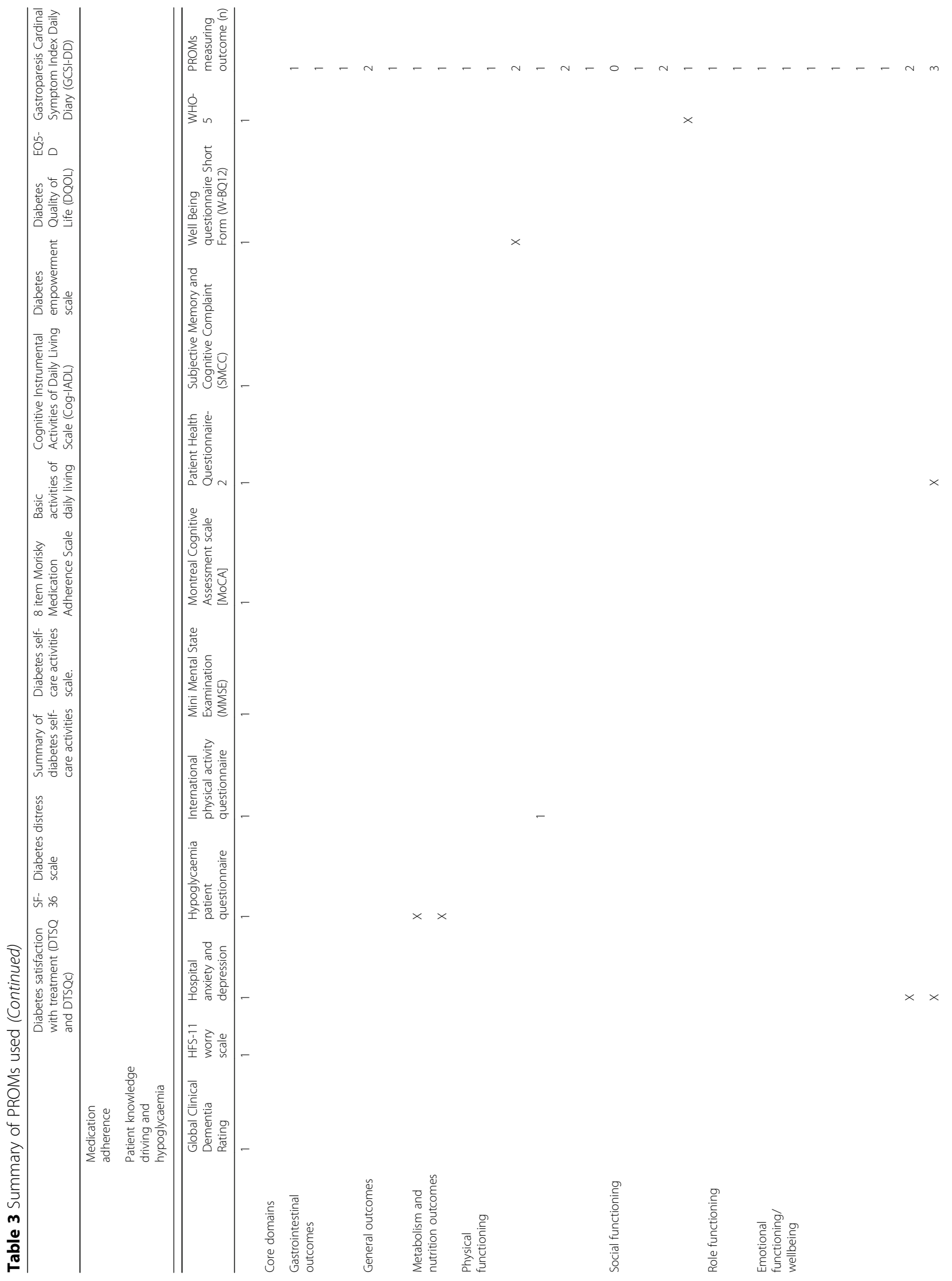




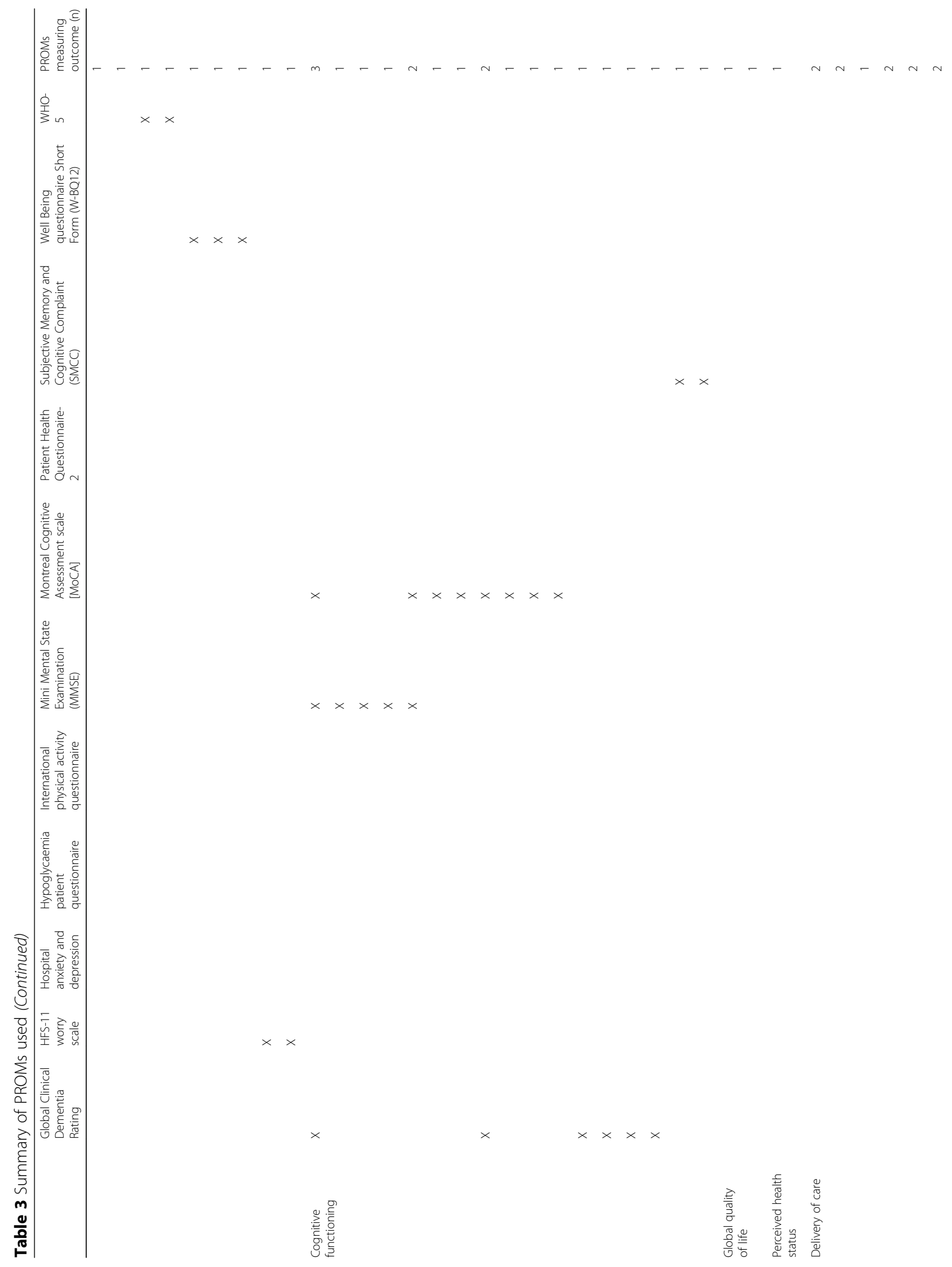




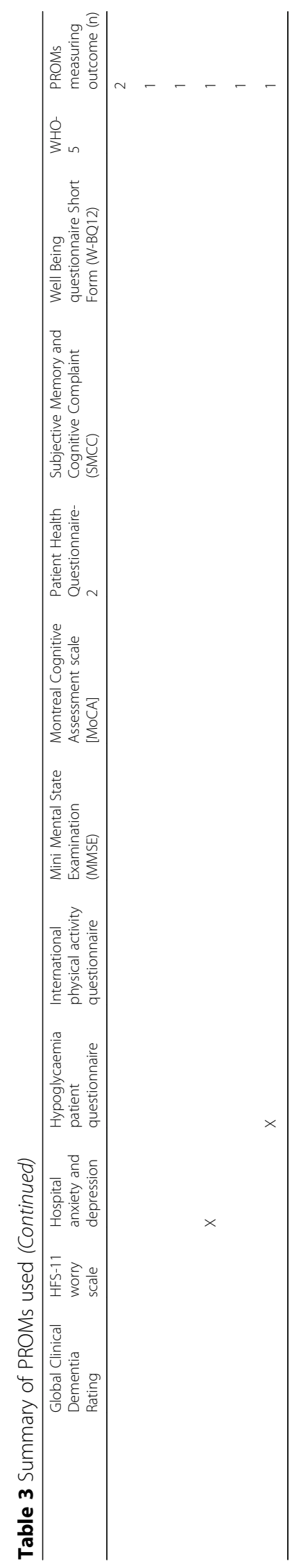


A limitation of the present study is that only one trials registry, clinicaltrials.gov, has been used. However, in the study by Gandhi et al., clinicaltrials.gov was the main registry source accounting for $81 \%$ of included studies [7]. In this study, only open (actively recruiting or will recruit in the near future) trials have been included, representing the current use of outcomes in trials treating hyperglycaemia in patients with type 2 diabetes mellitus. Including only open trials has the advantage that the outcomes used reflect the current state of affairs in a particular research area. In a topic area as vast as type 2 diabetes, this has additional importance of not only the resource needed to review studies and generate an outcomes list but also ensuring that the outcomes included in a subsequent Delphi survey are relevant and do not represent outdated and redundant outcomes.

A number of COSs exist for type 2 diabetes mellitus in clinical practice, but these too display heterogeneity in included outcomes [19]. The ICF COS [12] was developed using a consensus process and was designed for use in clinical practice although it has been suggested that the brief set of 28 items is suitable for use in clinical trials. However, the ICF set of 28 outcomes is impractical for use as a COS due to the large number of outcomes and the focus solely on function which may mean that it does not contain other outcomes important to patients with diabetes and health professionals caring for them.

This review of current registered trials highlights the need for a $\operatorname{COS}$ for use in clinical trials of type 2 diabetes; it will contribute to a preliminary list of outcomes and outcome domains for use in the first round of an online Delphi survey to identify which outcomes are of importance to researchers, healthcare professionals and patients.

\section{Additional files}

Additional file 1: Summary of diabetes research on COMET database. (DOCX $54 \mathrm{~kb}$ )

Additional file 2: List of all included studies. (XLSX $10 \mathrm{~kb}$ )

Additional file 3: Review of outcomes against the ICF core set. (DOCX $19 \mathrm{~kb}$ )

Additional file 4: ICF codes not used in outcomes. (XLSX 23 kb)

\begin{abstract}
Abbreviations
COMET: Core Outcome Measures in Effectiveness Trials; COS: Core outcome set; DPP-4: Dipeptidyl peptidase-4; DTSQ: Diabetes Treatment Satisfaction Questionnaire; GLP-1: Glucagon-like peptide 1; HbA1c: Glycated haemoglobin; ICF: International Classification of Functioning, Disability and Health; PROM: Patient-reported outcome measure; SGLT 2: Sodium-glucose co-transporter 2; WHO: World Health Organization
\end{abstract}

\section{Acknowledgements}

The SCORE-IT study team includes Serena Battaglia, Jacques Demotes-Mainard, Valerie Gailus-Durner, Silvio Garattini, Cecilia A C Prinsen, Michael Raess, Patricia da Silva-Buttkus, and Caroline B. Terwee.

\section{Funding}

This work has received funding from the European Union's Horizon 2020 research and innovation programme (CORBEL, under grant agreement no. 654248)

\section{Availability of data and materials}

The datasets used and/or analysed during the current study are available from the corresponding author on reasonable request. Data relating to included entries in the clinical trials.gov registry are provided in Additional file 1.

\section{Authors' contributions}

PRW, NH and RJ conceived and designed the study. $\mathrm{NH}, \mathrm{RJ}$ and PRW performed the search and extracted outcomes. NH, JW and PRW reviewed and categorised outcomes. NH drafted the manuscript; all authors reviewed and approved the manuscript. Collaborating authors, part of the SCORE-IT

Study Team: SB, J D-M, V G-D, SG, CACP, MR, PS-B, CBT.

Ethics approval and consent to participate

Not applicable.

\section{Consent for publication}

Not applicable.

\section{Competing interests}

PRW is member of the COMET Management Group and co-applicant on grants to support COMET and related work.

\section{Publisher's Note}

Springer Nature remains neutral with regard to jurisdictional claims in published maps and institutional affiliations.

\section{Author details}

${ }^{1}$ Department of Biostatistics, Institute of Translational Medicine, University of Liverpool, Liverpool L69 3GL, UK. ²Obesity and Endocrinology Clinical Research Group, Institute of Ageing and Chronic Disease, University Hospital Aintree, Longmoor Lane, Liverpool L9 7AL, UK.

Received: 15 September 2017 Accepted: 8 November 2017

Published online: 15 December 2017

\section{References}

1. Meigs JB, Muller DC, Nathan DM, Blake DR, Andres R. The natural history of progression from normal glucose tolerance to type 2 diabetes in the Baltimore longitudinal study of aging. Diabetes. 2003;52:1475-84.

2. American Diabetes Association. Diagnosis and classification of diabetes mellitus. Diabetes Care. 2010;33:S62-9.

3. Black C, Donnelly P, McIntyre L, Royle P, Shepherd JJ, Thomas S. Meglitinide analogues for type 2 diabetes mellitus. Cochrane Database Syst Rev. 2007;2:CD004654.

4. Vos RC, van Avendonk MJP, Jansen H, Goudswaard AN, van den Donk M, Gorter K, et al. Insulin monotherapy compared with the addition of oral glucose-lowering agents to insulin for people with type 2 diabetes already on insulin therapy and inadequate glycaemic control. Cochrane Database Syst Rev. 2016;9:CD006992.

5. Thomas D, Elliott EJ, Naughton GA. Exercise for type 2 diabetes mellitus. Cochrane Database Syst Rev. 2006:3:CD002968.

6. Goudswaard AN, Furlong NJ, Valk GD, Stolk RP, Rutten GEHM. Insulin monotherapy versus combinations of insulin with oral hypoglycaemic agents in patients with type 2 diabetes mellitus. Cochrane Database Syst Rev. 2004:4:CD003418.

7. Gandhi GY, Murad MH, Fujiyoshi A, Mullan RJ, Flynn DN, Elamin MB, et al. Patient-important outcomes in registered diabetes trials. JAMA 2008:299:2543-9.

8. Montori VM, Wang YG, Alonso-Coello P, Bhagra S. Systematic evaluation of the quality of randomized controlled trials in diabetes. Diabetes Care. 2006; 29:1833-8.

9. Heneghan C, Goldacre B, Mahtani KR. Why clinical trial outcomes fail to translate into benefits for patients. Trials. 2017;18:122. 
10. Tunis SR, Clarke M, Gorst SL, Gargon E, Blazeby JM, Altman DG, et al. Improving the relevance and consistency of outcomes in comparative effectiveness research. J Comp Eff Res. 2016;5:193-205.

11. ICF Core Sets for Diabetes Mellitus. https://www.icf-research-branch.org/icfcore-sets-projects2/cardiovascular-and-respiratory-conditions/icf-core-set-fordiabetes-mellitus.

12. Ruof J, Cieza A, Wolff B, Angst F, Ergeletzis D, Omar Z, et al. ICF Core Sets for diabetes mellitus. J Rehabil Med. 2004;(44 Suppl):100-6

13. Clarke M. Standardising outcomes for clinical trials and systematic reviews. Trials. 2007:8:39.

14. Wellard SJ, Cox H, Bhujoharry C. Issues in the provision of nursing care to people undergoing cardiac surgery who also have type 2 diabetes. Int J Nurs Pract. 2007;13:222-8.

15. Williamson PR, Altman DG, Bagley H, Barnes KL, Blazeby JM, Brookes ST, et al. The COMET Handbook: version 1.0. Trials. 2017:18:280

16. Reaney M, Elash CA, Litcher-Kelly L. Patient Reported Outcomes (PROs) used in recent Phase 3 trials for Type 2 Diabetes: A review of concepts assessed by these PROs and factors to consider when choosing a PRO for future trials. Diabetes Res Clin Pract. 2016;116:54-67.

17. Bradley C, Gamsu DS. Guidelines for encouraging psychological wellbeing: report of a Working Group of the World Health Organization Regional Office for Europe and International Diabetes Federation European Region St Vincent Declaration Action Program for Diabetes. Diabet Med. 1994;11:510-6.

18. Barsdorf Al, Rubinstein E, Jaksa A. Patient-reported outcomes (Pros) in diabetes clinical trials. Value Health. 2013;16:A168-9.

19. Mulcahy K, Maryniuk M, Peeples M, Peyrot M, Tomky D, Weaver T, et al. Diabetes self-management education core outcomes measures. Diabetes Educ. 2003;29:768-803.

\section{Submit your next manuscript to BioMed Central and we will help you at every step:}

- We accept pre-submission inquiries

- Our selector tool helps you to find the most relevant journal

- We provide round the clock customer support

- Convenient online submission

- Thorough peer review

- Inclusion in PubMed and all major indexing services

- Maximum visibility for your research

Submit your manuscript at www.biomedcentral.com/submit 